PROCEEDINGS OF THE

AMERICAN MATHEMATICAL SOCIETY

Volume 135, Number 7, July 2007, Pages 1959-1967

S 0002-9939(07)08629-7

Article electronically published on February 28, 2007

\title{
FRACTALIZED CYCLOTOMIC POLYNOMIALS
}

\author{
DAVID P. ROBERTS
}

(Communicated by Ken Ono)

\begin{abstract}
For each prime power $p^{m}$, we realize the classical cyclotomic polynomial $\Phi_{p^{m}}(x)$ as one of a collection of $3^{m}$ different polynomials in $\mathbf{Z}[x]$. We show that the new polynomials are similar to $\Phi_{p^{m}}(x)$ in many ways, including that their discriminants all have the form $\pm p^{c}$. We show also that the new polynomials are more complicated than $\Phi_{p^{m}}(x)$ in other ways, including that their complex roots are generally fractal in appearance.
\end{abstract}

\section{INTRODUCTION}

Cyclotomic polynomials $\Phi_{d}(x)$ and their associated number fields $\mathbf{Q}\left(e^{2 \pi i / d}\right)$ form a substantial topic in algebraic number theory, represented by the books [3] and 7. Here we restrict attention to the particularly interesting case where the index is a prime power, $d=p^{m}>1$. We realize

$$
\Phi_{p^{m}}(x)=\frac{x^{p^{m}}-1}{x^{p^{m-1}}-1}=\sum_{j=0}^{p-1} x^{j p^{m-1}}
$$

as one out of a collection of $3^{m}$ different polynomials in $\mathbf{Z}[x]$. Our new polynomials are denoted $\Phi_{p ; \tau_{1}, \ldots, \tau_{m}}(x)$, where the $\tau_{j}$ are chosen independently from the set $\{0,1, \infty\}$.

On the one hand, the $\Phi_{p ; \tau_{1}, \ldots, \tau_{m}}(x)$ are very similar to the classical $\Phi_{p^{m}}(x)=$ $\Phi_{p ; 1, \ldots, 1}(x)$. They have degree $\phi\left(p^{m}\right)=(p-1) p^{m-1}$, are irreducible over the integers, have polynomial discriminant of the form $\pm p^{c}$, field discriminant equal to their polynomial discriminant, and Galois group having size of the form $(p-1) p^{b}$. Our proofs of these facts follow classical proofs.

On the other hand, the $\Phi_{p ; \tau_{1}, \ldots, \tau_{m}}(x)$ are in general much more complicated than the classical $\Phi_{p^{m}}(x)$. Their roots are generally fractal in appearance, rather than intelligibly spaced on a circle. For $p=2$, there are often many real roots. The Galois groups $\operatorname{Gal}\left(\Phi_{p ; \tau_{1}, \ldots, \tau_{m}}(x)\right)$ are typically much larger than $\operatorname{Gal}\left(\Phi_{p^{m}}(x)\right)=\left(\mathbf{Z} / p^{m}\right)^{\times}$ and moreover highly nonabelian.

Section 2 defines our analogs. Section 3 proves the statements in the second paragraph. Section 4 communicates the complexity of our new polynomials; it draws some root plots, counts real roots, and computes some Galois groups.

For related material we refer the reader as follows. First, the complex roots of our fractalized cyclotomic polynomials $\Phi_{p ; \tau_{1}, \ldots, \tau_{m}}(x)$ are higher circular $p$-units in

Received by the editors November 2, 2005 and, in revised form, January 4, 2006

2000 Mathematics Subject Classification. Primary 11R21; Secondary 12E10, 37F99.

Key words and phrases. Cyclotomic polynomial, discriminant, fractal, Galois.

(C)2007 American Mathematical Society 
the sense of Anderson and Thara 2]. The general construction of [2] involves iterated covers of the Riemann sphere, $\hat{\mathbf{C}} \stackrel{f_{m}}{\rightarrow} \hat{\mathbf{C}}^{f_{m-1}} \rightarrow \cdots \stackrel{f_{2}}{\rightarrow} \hat{\mathbf{C}} \stackrel{f_{1}}{\rightarrow} \hat{\mathbf{C}}$, with each step $f_{j}$ conjugate by fractional linear transformations to $x \mapsto x^{p}$ and all critical values of $f_{1} \circ \cdots \circ f_{m}$ in $\{0,1, \infty\}$. We restrict the allowed fractional linear transformations to the six permuting the cusps $\{0,1, \infty\}$. Our restriction keeps each $f_{j}$ defined over Q; it keeps polynomial discriminants, not just field discriminants, of the form $\pm p^{c}$. Second, our polynomials illustrate the general technique of using iteration to construct infinite extensions of the rationals with only finitely many ramifying primes; [1] provides a general introduction to this technique. Third, our field discriminant formula provides a tool for studying higher ramification subgroups in the infinite extension of $\mathbf{Q}$ obtained by considering all our polynomials belonging to a given fixed prime at once; this application is pursued in the case $p=2$ in [6].

\section{Definition}

Let $p$ be a prime. Let $\mathbf{Z}[x]_{n}$ be the additive group of polynomials in $\mathbf{Z}[x]$ of degree $\leq n$. Define linear operators $R: \mathbf{Z}[x]_{n} \rightarrow \mathbf{Z}[x]_{n}$ and $F_{p}: \mathbf{Z}[x]_{n} \rightarrow \mathbf{Z}[x]_{p n}$ by

$$
\begin{aligned}
& (R f)(x)=(x-1)^{n} f\left(\frac{1}{1-x}\right), \\
& \left(F_{p} f\right)(x)=f\left(x^{p}\right) .
\end{aligned}
$$

We write $R$ instead of $R_{n}$ to lighten the notation. In all cases, the space $\mathbf{Z}[x]_{n}$ in which a given $f$ is to be considered will be clear; almost always, $n$ is simply the degree of $f$.

A fractalized cyclotomic polynomial for the prime $p$ is by definition any polynomial obtained from $\Phi_{p}(x) \in \mathbf{Z}[x]_{p-1}$ by successive application of $F_{p}$ and $R$ in any order. Note that $\left(F_{p}^{m-1} \Phi_{p}\right)(x)=\Phi_{p}\left(x^{p^{m-1}}\right)$ is just the classical cyclotomic polynomial $\Phi_{p^{m}}(x)$. Inserting $R$ 's among the $F_{p}$ 's "fractures" this construction process; this is one reason we use the word "fractalized."

Next, we give a unique name to each fractalized cyclotomic polynomial. As a first step, note that $R$ is related to the map $g_{(01 \infty)}: \hat{\mathbf{C}} \rightarrow \hat{\mathbf{C}}: x \mapsto 1 /(1-x)$. The map $g_{(01 \infty)}$ rotates the cusps of $\hat{\mathbf{C}}$, in the sense that $0 \mapsto 1,1 \mapsto \infty$, and $\infty \mapsto 0$. One can check that signs have been chosen properly in (2.1) so that $R$, like $g_{(01 \infty)}$, has order three. Thus, using $R$ alone, one can only construct three polynomials from $\Phi_{p}(x)$. We name them as follows:

$$
\begin{aligned}
\Phi_{p ; 0}(x) & =\left(R \Phi_{p}\right)(x)=(-1)^{p-1} \sum_{j=0}^{p-1}\left(\begin{array}{c}
p \\
j+1
\end{array}\right)(-x)^{j}, \\
\Phi_{p ; 1}(x) & =\Phi_{p}(x)=\sum_{j=0}^{p-1} x^{j} \\
\Phi_{p ; \infty}(x) & =\left(R^{-1} \Phi_{p}\right)(x)=\sum_{j=0}^{p-1}\left(\begin{array}{c}
p \\
j
\end{array}\right)(-x)^{j} .
\end{aligned}
$$

Passing from the summation in (2.4) to the summations in (2.3) and (2.5) is a simple classical computation centering on expanding $(x-1)^{p}$ by the binomial theorem.

As the second and main step, we bring in $F_{p}$. Note that the operator $F_{p}$ is related to the map $f_{p}=\hat{\mathbf{C}} \rightarrow \hat{\mathbf{C}}: x \mapsto x^{p}$. To treat 0,1 , and $\infty$ on an equal 
footing, we define degree $p$ maps

$$
\begin{aligned}
f_{p ; 0} & =g_{(01 \infty)}^{-1} f_{p} g_{(01 \infty)}: \quad x \mapsto 1-(1-x)^{p}, \\
f_{p ; 1} & =f_{p}: \quad x \mapsto x^{p}, \\
f_{p ; \infty} & =g_{(01 \infty)} f_{p} g_{(01 \infty)}^{-1}: \quad x \mapsto \frac{x^{p}}{x^{p}-(x-1)^{p}} .
\end{aligned}
$$

All three maps fix 0,1 , and $\infty$. The critical points of $f_{p ; \tau}$ are the two points of $\{0,1, \infty\}-\{\tau\}$, each with multiplicity $p-1$. Motivated by this discussion of rational functions, we define

$$
\begin{aligned}
F_{p ; 0} & =R F_{p} R^{-1}, \\
F_{p ; 1} & =F_{p}, \\
F_{p ; \infty} & =R^{-1} F_{p} R .
\end{aligned}
$$

Henceforth we will emphasize the three operators $F_{p ; \tau}$, rather than $F_{p}$ and $R$.

Definition 2.1. Let $p$ be a prime, $m$ a positive integer, and $\tau_{1}, \ldots, \tau_{m}$ elements of $\{0,1, \infty\}$. Then the corresponding fractalized cyclotomic polynomial $\Phi_{p ; \tau_{1}, \ldots, \tau_{m}}(x)$ is defined by (2.3), (2.4), (2.5) for $m=1$ and by

$$
\Phi_{p ; \tau_{1}, \ldots, \tau_{m}}=F_{p ; \tau_{m}} \cdots F_{p ; \tau_{2}} \Phi_{p ; \tau_{1}}
$$

for $m \geq 2$.

By construction, $\Phi_{p ; \tau_{1}, \ldots, \tau_{m}}(x)$ lies in the space $\mathbf{Z}[x]_{\phi\left(p^{m}\right)}$. Clearly, the polynomials $\Phi_{p ; \tau_{1}, \ldots, \tau_{m}}(x)$ just defined exhaust the fractalized cyclotomic polynomials: one cannot get any other polynomials by applying $R$ and $F_{p}$ iteratively to $\Phi_{p}(x)$.

There is a basic symmetry among the fractalized cyclotomic polynomials which we have not yet fully incorporated into our formalism. Define $S: \mathbf{Z}[x]_{n} \rightarrow \mathbf{Z}[x]_{n}$ by $(S f)(x)=x^{n} f(1 / x)$. Then $S$ is related to the rational map $g_{(0 \infty)}: \hat{\mathbf{C}} \rightarrow \hat{\mathbf{C}}: x \mapsto$ $1 / x$. In fact, the association $(0 \infty) \mapsto g_{(0 \infty)}^{*}=S$ and $(01 \infty) \mapsto g_{(01 \infty)}^{*}=R$ extends to a homomorphism $\alpha \mapsto g_{\alpha}^{*}$ from $S_{3}$ to operators on $\mathbf{Z}[x]_{n}$. The basic symmetry is then

$$
g_{\alpha}^{*} \Phi_{p ; \tau_{1}, \ldots, \tau_{m}}=\Phi_{p ; \alpha^{-1} \tau_{1}, \ldots, \alpha^{-1} \tau_{m}} .
$$

Thus the $3^{m}$ different $\Phi_{p ; \tau_{1}, \ldots, \tau_{m}}(x)$ fall into the $S_{3}$-orbit of $\Phi_{p^{m}}(x)=\Phi_{p ; 1, \ldots, 1}(x)$, which contains three polynomials, and $\left(3^{m}-3\right) / 6$ more $S_{3}$-orbits, each with six polynomials.

\section{Similarities}

The theorem of this paper gives various properties of the fractalized cyclotomic polynomials. All of them are standard in the case of the cyclotomic polynomial $\Phi_{p^{m}}(x)=\Phi_{p ; 1, \ldots, 1}(x)$. The proofs in the general case consist mostly of applying standard general facts to our situation. To treat $\infty$ on the same footing as 0 and 1 , we define $f(\infty)=(S f)(0)=a_{0}$ for $f(x)=a_{0} x^{n}+\cdots \in \mathbf{Z}[x]_{n}$.

Theorem 3.1. Let $p$ be a prime, $m$ a positive integer, and $\tau_{1}, \ldots, \tau_{m}$ elements of $\{0,1, \infty\}$. Let $\Phi_{p ; \tau_{1}, \ldots, \tau_{m}}(x)$ be the corresponding fractalized cyclotomic polynomial. Then 
(Cuspidal values) For $\sigma \in\{0,1, \infty\}$ one has

$$
\Phi_{p ; \tau_{1}, \ldots, \tau_{m}}(\sigma)= \begin{cases} \pm p & \text { if } \sigma=\tau_{1}, \\ \pm 1 & \text { if } \sigma \neq \tau_{1},\end{cases}
$$

with all signs positive if $p$ is odd. In particular, $\Phi_{p ; \tau_{1}, \ldots, \tau_{m}}(x)$ has degree $\phi\left(p^{m}\right)$.

(Reduction modulo $p$ ) Let $\Psi_{0}(x)=x, \Psi_{1}(x)=-x+1$, and $\Psi_{\infty}(x)=-1$. Then

$$
\Phi_{p ; \tau_{1}, \ldots, \tau_{m}}(x) \equiv \Psi_{\tau_{1}}(x)^{\phi\left(p^{m}\right)} \text { modulo } p .
$$

(Irreducibility) $\Phi_{p ; \tau_{1}, \ldots, \tau_{m}}$ is irreducible in $\mathbf{Z}[x]$.

(Polynomial discriminant) The polynomial discriminant of $\Phi_{p ; \tau_{1}, \ldots, \tau_{m}}(x)$ is $D\left(\Phi_{p ; \tau_{1}, \ldots, \tau_{m}}(x)\right)= \pm p^{c}$ with

$$
c=p-2+\sum_{j=2}^{m}(p-1)^{2} p^{j-2} j+\sum_{j=2}^{m} \delta\left(\tau_{1} \neq \tau_{j}\right)(p-1) p^{m-j} .
$$

Here $\delta\left(\tau_{1} \neq \tau_{j}\right)$ is 1 if $\tau_{1}$ and $\tau_{j}$ are different, and otherwise 0 .

(Field discriminant) The field discriminant of $\mathbf{Q}[x] / \Phi_{p ; \tau_{1}, \ldots, \tau_{m}}(x)$ is

$$
d\left(\Phi_{p ; \tau_{1}, \ldots, \tau_{m}}(x)\right)=D\left(\Phi_{p ; \tau_{1}, \ldots, \tau_{m}}(x)\right) .
$$

(Galois group order) The Galois group of $\Phi_{p ; \tau_{1}, \ldots, \tau_{m}}(x)$ has order of the form $(p-1) p^{b}$.

Proof. Cuspidal values. By inspection of (2.3), (2.4), and (2.5), one sees that (3.1) holds when $m=1$. In general, our operators preserve cuspidal values up to sign as follows. First, by inspection one has

$$
(R f)(0)=(-1)^{n} f(1), \quad(R f)(1)=(-1)^{n} f(\infty), \quad(R f)(\infty)=f(0) .
$$

Second, clearly $\left(F_{p, 1} f\right)(\sigma)=\sigma$, for all $\sigma=\{0,1, \infty\}$. Using $F_{p, 0}=R F_{p, 1} R^{-1}$ and $F_{p, \infty}=R^{-1} F_{p, 1} R^{-1}$ one finds

$$
\left(F_{p ; \tau} f\right)(\sigma)=\left\{\begin{aligned}
(-1)^{(p-1) n} f(\sigma) & \text { if }(\tau, \sigma)=(0,0),(0,1),(\infty, 1),(\infty, \infty), \\
f(\sigma) & \text { if } \sigma \neq \tau .
\end{aligned}\right.
$$

Here the factor $(-1)^{(p-1) n}$ arises in the first case when a sign $(-1)^{n}$ enters before the application of $F_{p}$ and then a second sign $(-1)^{p n}$ enters after the application of $F_{p}$. By induction, (3.1) holds for general $m$, with all signs positive if $p$ is odd.

Reduction modulo $p$. By inspection of (2.3), (2.4), and (2.5), one sees that the congruence (3.2) holds for $m=1$. Working in $\mathbf{F}_{p}[x]$ rather than $\mathbf{Z}[x]$, and considering $\Psi_{\tau}^{n} \in \mathbf{F}_{p}[x]_{n}$, one has the general formulas $R \Psi_{\tau}^{n}=\Psi_{(0 \infty 1) \tau}^{n}$ and $F_{p} \Psi_{\tau}^{n}=$ $\Psi_{\tau}^{p n}$. These two formulas prove the congruence (3.2) for general $m$ by induction.

Irreducibility. A polynomial $f(x)=a_{0} x^{n}+\cdots+a_{n}$ in $\mathbf{Z}[x]_{n}$ is called a $p$ Eisenstein polynomial if and only if

$$
\begin{aligned}
\operatorname{ord}_{p}\left(a_{0}\right) & =0 \\
\operatorname{ord}_{p}\left(a_{i}\right) & \geq 1(1 \leq i \leq n-1) \\
\operatorname{ord}_{p}\left(a_{n}\right) & =1
\end{aligned}
$$

A general fact about $p$-Eisenstein polynomials is that they are irreducible in $\mathbf{Z}[x]$, because they are even irreducible in $\mathbf{Z}_{p}[x]$, where $\mathbf{Z}_{p}$ is the ring of $p$-adic integers. If $\tau_{1}=0$, then $\Phi_{p ; \tau_{1}, \ldots, \tau_{m}}(x)$ is $p$-Eisenstein by the results on cuspidal values and 
reduction modulo $p$, hence irreducible. Irreducibility in the case $\tau_{1} \neq 0$ then follows by transformation to the $\tau_{1}=0$ case, via (2.13).

Polynomial discriminant. In general, for $f(x)=a_{0} x^{n}+\cdots \in \mathbf{Z}[x]_{n}$ with roots $\alpha_{1}, \ldots, \alpha_{n} \in \mathbf{C}$, its discriminant is

$$
D(f)=a_{0}^{2 n-2} \prod_{i<j}\left(\alpha_{i}-\alpha_{j}\right)^{2} .
$$

Standard facts about discriminants include equivariance with respect to fractional linear transformations. In particular,

$$
D\left(g_{\alpha}^{*} f\right)=D(f)
$$

for $\alpha \in S_{3}$. Equality (3.7) can be proved directly from the definition (3.6) for the cases $\alpha=(01)$ and $\alpha=(0 \infty)$, and the case of general $\alpha$, including $R=g_{(01 \infty)}^{*}$ then follows. Likewise, a standard fact is $D\left(F_{p} f\right)= \pm p^{n p} f(0)^{p-1} f(\infty)^{p-1} D(f)^{p}$. Combining this fact with (3.4) and (3.7) gives

$$
D\left(F_{p ; \tau} f\right)= \pm p^{n p} f\left(\tau^{\prime}\right)^{p-1} f\left(\tau^{\prime \prime}\right)^{p-1} D(f)^{p},
$$

where $\left\{\tau, \tau^{\prime}, \tau^{\prime \prime}\right\}=\{0,1, \infty\}$. For $m=1$, Equation (3.3) reduces to the known formula $D\left(\Phi_{p}\right)= \pm p^{p-2}$. The general case of (3.3) then follows from induction, using (2.12) and (3.8). In particular, in the expression for $D\left(F_{p ; \tau_{j}} f\right)$ given by (3.8) with $f=\Phi_{p ; \tau_{1}, \ldots, \tau_{j-1}}$, one has $\left\{f\left(\tau^{\prime}\right), f\left(\tau^{\prime \prime}\right)\right\}=\{ \pm 1, \pm 1\}$ if $\tau_{j}=\tau_{1}$ and $\left\{f\left(\tau^{\prime}\right), f\left(\tau^{\prime \prime}\right)\right\}=\{ \pm 1, \pm p\}$ if $\tau_{j} \neq \tau_{1}$, by (3.1). In the latter case one gets a contribution of $p^{p-1}$ to $D\left(F_{p ; \tau_{j}} f\right)$. In each of the remaining $m-j$ applications of (3.8), this contribution accumulates in the $D(f)$ factor on the right of (3.8), being raised to the $p^{\text {th }}$ power each time. This accounts for the term $\delta\left(\tau_{1} \neq \tau_{j}\right)(p-1) p^{m-j}$ in (3.3).

Field discriminant. In general, an irreducible polynomial $f(x) \in \mathbf{Z}[x]$ determines a number field $\mathbf{Q}[x] / f(x)$. The discriminant $D(f) \in \mathbf{Z}$ of the polynomial and the discriminant $d(f) \in \mathbf{Z}$ of the field are related by $d(f)=D(f) / i(f)^{2}$, for $i(f)$ a positive integer. Also, in general, if $f(x)$ is a $p$-Eisenstein polynomial, then $\operatorname{ord}_{p}(i(f))=0$. These generalities apply directly to $\Phi_{p ; \tau_{1}, \ldots, \tau_{m}}(x)$ when $\tau_{1}=0$ and, since $D\left(\Phi_{p ; \tau_{1}, \ldots, \tau_{m}}(x)\right)$ has the form $\pm p^{c}$, yield the equality of the polynomial and field discriminants. Equality in the case $\tau_{1} \neq 0$ then follows by transformation to the $\tau_{1}=0$ case, via (2.13).

Galois group size. Let $\alpha_{m}$ be a complex root of $\Phi_{p ; \tau_{1}, \ldots, \tau_{m}}(x)$. Inductively define $\alpha_{m-1}, \ldots, \alpha_{1}$ by $\alpha_{j-1}=f_{p ; \tau_{j}}\left(\alpha_{j}\right)$ so that $\alpha_{j}$ is a root of $\Phi_{p ; \tau_{1}, \ldots, \tau_{j}}(x)$. In $\mathbf{C}$, we have the tower of fields $K_{j}=\mathbf{Q}\left(\alpha_{1}, \ldots, \alpha_{j}\right)$. We have $K_{1}=\mathbf{Q}\left(e^{2 \pi i / p}\right)$ and the $p^{\text {th }}$ power of either $\alpha_{j}, g_{(01 \infty)}\left(\alpha_{j}\right)$, or $g_{(01 \infty)}^{-1}\left(\alpha_{j}\right)$ is in $K_{j-1}$. By Kummer theory, for $j=2, \ldots, m$, the extension $K_{j} / K_{j-1}$ is Galois with group $\mathbf{Z} / p$. Let $K_{j}^{g}$ be the Galois closure of $K_{j}$. Our discussion shows that each $K_{j}^{g} / K_{1}$ is Galois with group of order a power of $p$, and so the Galois group $\operatorname{Gal}\left(\Phi_{p ; \tau_{1}, \ldots, \tau_{m}}(x)\right)=\operatorname{Gal}\left(K_{m}^{g} / \mathbf{Q}\right)$ has order of the form $(p-1) p^{b}$.

\section{Differences}

Root plots. The set $X_{p ; \tau_{1}, \ldots, \tau_{m}}$ of complex roots of any $\Phi_{p ; \tau_{1}, \ldots, \tau_{m}}(x)$ is easily obtained by iteratively applying the formulas

$$
\begin{aligned}
X_{p ; \tau_{1}} & =f_{p ; \tau_{1}}^{-1}\left(\tau_{1}\right)-\left\{\tau_{1}\right\}, \\
X_{p ; \tau_{1}, \ldots, \tau_{m}} & =f_{p ; \tau_{m}}^{-1}\left(X_{p ; \tau_{1}, \ldots, \tau_{m-1}}\right) \quad(m \geq 2) .
\end{aligned}
$$


Explicitly, the inverse image operators are given by

$$
\begin{aligned}
f_{p ; 0}^{-1}(x) & =\left\{1-\epsilon(1-x)^{1 / p}\right\} \\
f_{p ; 1}^{-1}(x) & =\left\{\epsilon x^{1 / p}\right\} \\
f_{p ; \infty}^{-1}(x) & =\left\{\frac{1}{1-\epsilon(1-1 / x)^{1 / p}}\right\}
\end{aligned}
$$

with $\epsilon$ running over the $p^{\text {th }}$ roots of unity in each case.

If the last $k$ indices $\tau_{m-k+1}, \ldots, \tau_{m}$ are all the same, then the root set $X_{p ; \tau_{1}, \ldots, \tau_{m}}$ is stable under a group $\mu_{p^{k}, \tau_{m}}$ of $p^{k}$ fractional linear transformations. These transformations take the nicest algebraic form when $\tau_{m}=1$, as then they are given by multiplication by $p^{k \text { th }}$ roots of unity. Independently, $X_{p ; \tau_{1}, \ldots, \tau_{m}}$ is invariant under complex conjugation $\sigma$. All together, $X_{p ; \tau_{1}, \ldots, \tau_{m}}$ is stable under a dihedral group $\mu_{p^{k}, \tau_{m}} \rtimes\{1, \sigma\}$.

Figure 4.1 shows the roots of three fractalized cyclotomic polynomials with $p=2$. Figure 4.2 likewise shows the roots of two fractalized cyclotomic polynomials with $p=3$. In each case we have taken $\tau_{m}=1$ and $k=1$, so the symmetry groups have order 4 and 6 respectively and are clearly visible. The top left image in Figure 4.1 appears commonly in the popular fractal literature, as it approximates the Julia set for the quadratic polynomial $x^{2}-1$.

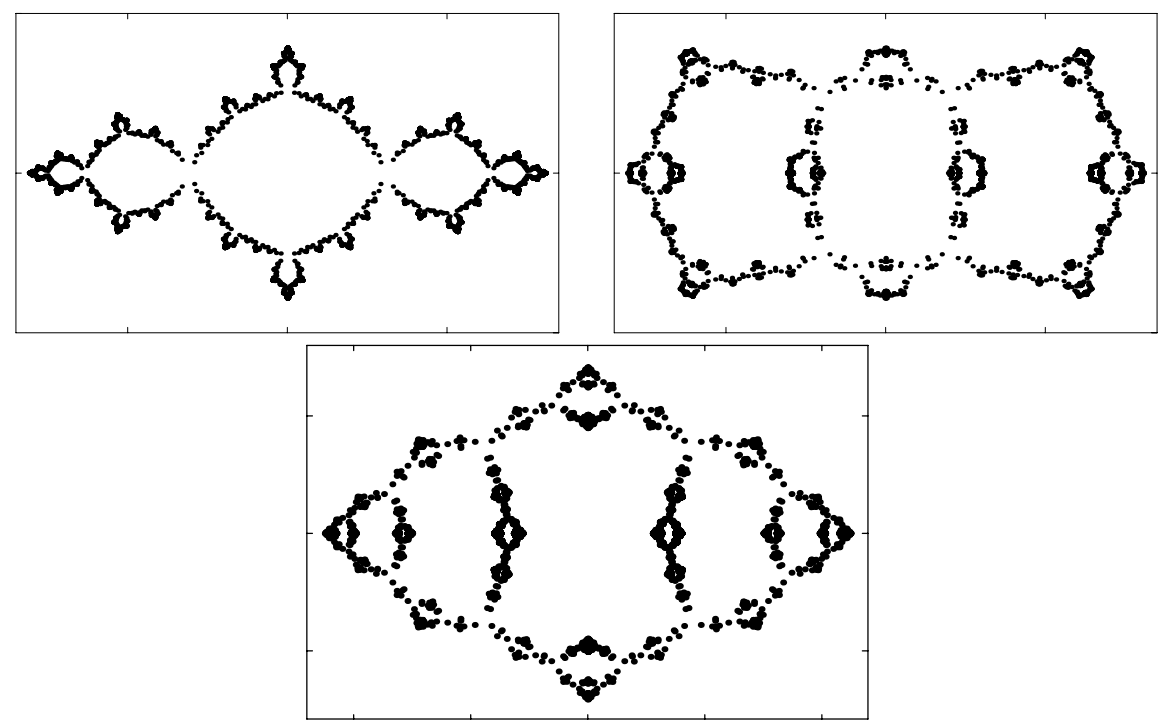

Figure 4.1. Roots of $\Phi_{2 ; 1,0,1,0,1,0,1,0,1,0,1,0,1}(x)$ on the top left and $\Phi_{2 ; 1,0, \infty, 0,1,0, \infty, 0,1,0, \infty, 0,1}(x)$ on the top right, each in the window $[-1.7,1.7] \times[-1,1]$ of the complex plane. Roots of $\Phi_{2 ; 1,0, \infty, 1,0, \infty, 1,0, \infty, 1,0, \infty, 1}(x)$ on the bottom, drawn to the same scale, now in the larger window $[-2.4,2.4] \times[-1.6,1.6]$. There are 4096 roots in each case, with the number of real roots being respectively 2,338 , and 466 . 

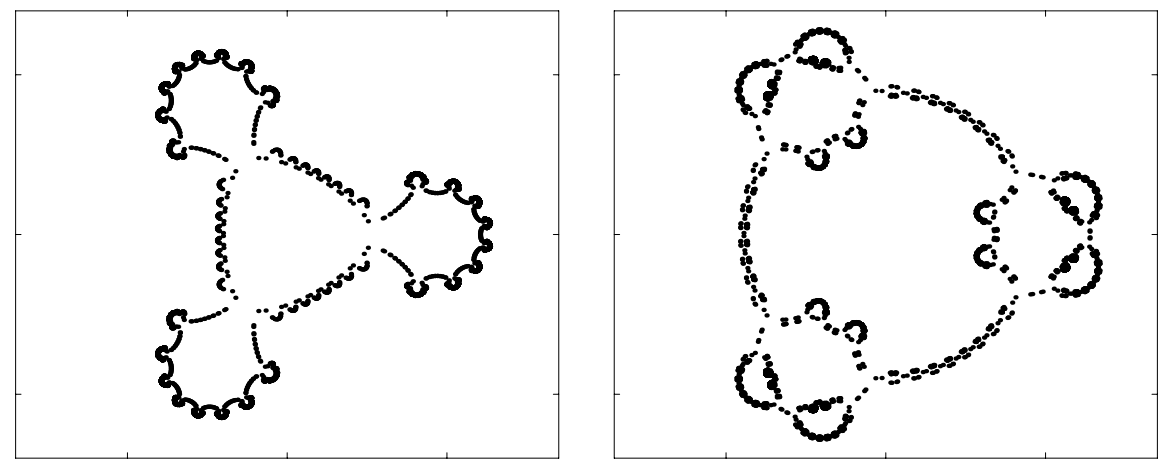

Figure 4.2. Roots of $\Phi_{3 ; 1,0,0,1,1,0,0,1}(x)$ on the left and $\Phi_{3 ; 1,0, \infty, 1,1, \infty, 0,1}(x)$ on the right, each drawn in the window $[-1.7,1.7] \times[-1.4,1.4]$, using the same scale as Figure 4.1] Both polynomials here have $\phi\left(3^{8}\right)=2 \cdot 3^{7}=4374$ roots, all nonreal.

Real roots. For $m \geq 2$, the roots of $\Phi_{p ; \tau_{1}, \ldots, \tau_{m}}(x)$ map $p$-to- 1 to the roots of $\Phi_{p ; \tau_{1}, \ldots, \tau_{m-1}}(x)$ under $f_{p ; \tau_{m}}$. The mapping is $\operatorname{Gal}(\overline{\mathbf{Q}} / \mathbf{Q})$-equivariant and in particular $\operatorname{Gal}(\mathbf{C} / \mathbf{R})$-equivariant. Thus a root $\alpha$ can be real only if $f_{p ; \tau_{m}}(\alpha)$ is real. If $p$ is odd, then no $\Phi_{p ; \tau_{1}, \ldots, \tau_{m}}(x)$ has real roots simply because no $\Phi_{p ; \tau_{1}}(x)$ has real roots. Henceforth, we restrict to $p=2$ and derive a simple recursive formula for $s_{\tau_{1}, \ldots, \tau_{m}}$, the number of real roots of $\Phi_{2 ; \tau_{1}, \ldots, \tau_{m}}(x)$.

Consider the real circle $\hat{\mathbf{R}}=\mathbf{R} \cup\{\infty\}$ in the Riemann sphere $\hat{\mathbf{C}}$. For $\sigma \in$ $\{0,1, \infty\}$, let $I_{\sigma}$ be the component of $\hat{\mathbf{R}}-\{0,1, \infty\}$ which does not have $\sigma$ in its closure. Thus $I_{0}=(1, \infty), I_{1}=(-\infty, 0)$, and $I_{\infty}=(0,1)$. For $\sigma \in\{0,1, \infty\}$, let $s_{\tau_{1}, \ldots, \tau_{m}}(\sigma)$ be the number of roots of $\Phi_{2 ; \tau_{1}, \ldots, \tau_{m}}(x)$ in the interval $I_{\sigma}$. Clearly,

$$
s_{\tau_{1}, \ldots, \tau_{m}}=\sum_{\sigma \in\{0,1, \infty\}} s_{\tau_{1}, \ldots, \tau_{m}}(\sigma) .
$$

In the case $m=1$, we have $\Phi_{2 ; 0}(x)=x-2, \Phi_{2 ; 1}(x)=x+1$, and $\Phi_{2 ; \infty}(x)=-2 x+1$. Thus

$$
s_{\tau_{1}}(\sigma)= \begin{cases}1 & \text { if } \sigma=\tau_{1} \\ 0 & \text { if } \sigma \neq \tau_{1}\end{cases}
$$

So (4.1) recovers the simple fact that $s_{\tau_{1}}=1$ in all three cases.

Under the map $f_{2 ; \tau}: \hat{\mathbf{R}} \rightarrow \hat{\mathbf{R}}$, an element in $I_{\tau}$ has no preimages. For $\sigma \neq \tau$, an element in $I_{\sigma}$ has two preimages, one in $I_{\sigma}$ and the other in $I_{\tau}$. These statements are completely clear in the case of $f_{2 ; 1}$ and then follow for the other two maps. Our discussion proves the following result.

Proposition 4.1. The number $s_{\tau_{1}, \ldots, \tau_{m}}$ of real roots of $\Phi_{2 ; \tau_{1}, \ldots, \tau_{m}}(x)$ is given by (4.1), (4.2), and the recursion

$$
s_{\tau_{1}, \ldots, \tau_{m}}(\sigma)= \begin{cases}s_{\tau_{1}, \ldots, \tau_{m-1}}\left(\sigma^{\prime}\right)+s_{\tau_{1}, \ldots, \tau_{m-1}}\left(\sigma^{\prime \prime}\right) & \text { if } \sigma=\tau_{m}, \\ s_{\tau_{1}, \ldots, \tau_{m-1}}(\sigma) & \text { if } \sigma \neq \tau_{m},\end{cases}
$$

with $\left\{\sigma, \sigma^{\prime}, \sigma^{\prime \prime}\right\}=\{0,1, \infty\}$. 
One can be completely explicit about $s_{\tau_{1}, \ldots, \tau_{m}}$ in three special cases as follows. First, note that if $\tau_{1}=\tau_{2}$, then already all three $s_{\tau_{1}, \tau_{1}}(\sigma)$ are 0 , giving the general formula $s_{\tau_{1}, \tau_{1}, \tau_{3}, \ldots, \tau_{m}}=0$. Second, if $\tau_{1} \neq \tau_{2}$ and all the other $\tau_{3}, \ldots, \tau_{m}$ are in $\left\{\tau_{1}, \tau_{2}\right\}$, then inductively one has $s_{\tau_{1}, \ldots, \tau_{m}}=2$. This case is represented by the upper left plot in Figure 4.1. Third, suppose that $\tau_{1} \neq \tau_{2}$ and each index $\tau_{3}, \ldots$, $\tau_{m}$ is taken to be the unique index different from its two immediate predecessors. Then inductively for $m \geq 2$ one has $s_{\tau_{1}, \ldots, \tau_{m}}=2 s_{\tau_{1}, \ldots, \tau_{m}}\left(\tau_{m}\right)=2 F_{m}$, where the $F_{m}$ is the $m^{\text {th }}$ Fibonacci number.

In fact, Fibonacci-like behavior can be seen in the root plot of any $\Phi_{2 ; \tau_{1}, \ldots, \tau_{m}}(x)$ having real roots. For example, if $\tau_{m}=1$, then by the $x \mapsto-x$ symmetry, the number of roots in $(-\infty, 0)$ is equal to the number of roots in $(0,1)$ plus the number of roots in $(1, \infty)$. In the three plots in Figure 4.1, this equation is $1=0+1$, $169=70+99$, and $233=144+89$.

Galois groups. The Galois group of $\Phi_{p^{m}}(x)$ is the group $\left(\mathbf{Z} / p^{m}\right)^{\times}$, of order $\phi\left(p^{m}\right)=(p-1) p^{m-1}$. While in general $G=\operatorname{Gal}\left(\Phi_{p ; \tau_{1}, \ldots, \tau_{m}}(x)\right)$ also has order of the form $(p-1) p^{b}$, computation shows that the isomorphism type of $G$ and even its order can depend subtly on the indices $\tau_{1}, \ldots, \tau_{m}$.

General theorems give one some control over the possibilities for $G$. For example, for $p=2$ the result of 4 says that a finite Galois 2-extension of $\mathbf{Q}$ ramified at 2 only has Galois group generated by complex conjugation and a single other element. So while the Sylow 2-subgroup $P$ of $S_{2^{m-1}}$ has size $2^{2^{m-1}-1}$, any $\operatorname{Gal}\left(\Phi_{2 ; \tau_{1}, \ldots, \tau_{m}}(x)\right)$ must be smaller when $m \geq 4$, as $P$ requires $m-1$ elements to generate it.

TABLE 4.1. Galois groups $G=\operatorname{Gal}\left(\Phi_{2 ; \tau_{1}, \ldots, \tau_{4}}(x)\right)$, with $a, b$, and $c$ representing distinct elements of $\{0,1, \infty\}$.

\begin{tabular}{|ccl||ccl|}
\hline$\tau_{1} \tau_{2} \tau_{3} \tau_{4}$ & $\operatorname{ord}_{2}(|G|)$ & $G$ & $\tau_{1} \tau_{2} \tau_{3} \tau_{4}$ & $\operatorname{ord}_{2}(|G|)$ & $G$ \\
\hline$a a a a$ & 3 & $T 2$ & $a b a c$ & 6 & $T 28$ \\
$a a a b$ & 5 & $T 21$ & $a b b a$ & 6 & $T 30$ \\
$a a b a$ & 5 & $T 19$ & $a b b b$ & 4 & $T 8$ \\
$a a b b$ & 5 & $T 17$ & $a b b c$ & 6 & $T 28$ \\
$a a b c$ & 4 & $T 6$ & $a b c a$ & 6 & $T 27$ \\
$a b a a$ & 4 & $T 8$ & $a b c b$ & 6 & $T 27$ \\
$a b a b$ & 6 & $T 30$ & $a b c c$ & 5 & $T 16$ \\
\hline
\end{tabular}

Table 4.1presents all possibilities for the case $(p, m)=(2,4)$ of octic polynomials. Galois groups were computed using [5], with $T n$ indicating that $G$ is the $n^{\text {th }}$ group on the standard list of fifty octic groups. A much more elaborate computation for $(p, m)=(2,5)$ found that while $\operatorname{ord}_{2}(|G|)$ is 4 in the cyclotomic case, it ranges from 6 to 11 in the forty other cases, with in total twenty-eight isomorphism classes of degree 16 permutation groups $G$ represented. This situation for general $(p, m)$ is quite unusual for Galois theory, as one has a collection of explicit polynomials but no generic expectation for their Galois groups. 


\section{REFERENCES}

[1] Wayne Aitken, Farhsid Hajir, Christian Maire, Finitely ramified iterated extensions. Int. Math. Res. Not. 2005, no. 14, 855-880. MR2146860 (2006c:11125)

[2] Greg W. Anderson, Yasutaka Ihara, Pro- $\ell$ branching coverings of $\mathbf{P}^{1}$ and higher circular

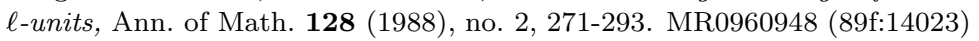

[3] Serge Lang, Cyclotomic fields I and II. Combined second edition. With an appendix by Karl Rubin, Graduate Texts in Mathematics, 121, Springer-Verlag, New York, 1990. xviii + 433pp. MR1029028 (91c:11001)

[4] G. N. Markšaĭtis, On p-extensions with one critical number (Russian), Izv. Akad. Nauk SSSR Ser. Mat. 27 (1963), 463-466. MR0151452 (27:1437)

[5] PARI/GP, Version 2.1.5, Bordeaux, 2004, http://pari.math.u-bordeaux.fr/.

[6] David P. Roberts, 2-adic ramification in some 2-extensions of $\mathbf{Q}$, in preparation.

[7] Lawrence C. Washington, Introduction to cyclotomic fields. Second edition, Graduate Texts in Mathematics, 83, Springer-Verlag, New York, 1997. xiv + 487pp. MR1421575 (97h:11130)

Division of Science and Mathematics, University of Minnesota-Morris, Morris, MinNESOTA 56267

E-mail address: roberts@morris.umn.edu 